\title{
Effects of probiotics on growth performance, blood parameters, and antibody stimulation in piglets
}

\author{
Z.C. Dlamini ${ }^{1,2}$, R.L.S. Langa ${ }^{1}$, O. A. Aiyegoro ${ }^{1,2, \#}$ \& A.I. $\mathrm{Okoh}^{2}$ \\ ${ }^{1}$ Agricultural Research Council - Animal Production Institute, Gastrointestinal Microbiology and Biotechnology Division, \\ Irene 0062, South Africa \\ ${ }^{2}$ SAMRC Microbial Water Quality Monitoring Centre, University of Fort Hare, Alice, 5700, South Africa
}

(Received 1 June 2017; Accepted 4 August 2017; First published online 25 September 2017)

\author{
Copyright resides with the authors in terms of the Creative Commons Attribution 4.0 South African License. \\ See: http://creativecommons.org/licenses/by/4.0/za \\ Condition of use: The user may copy, distribute, transmit and adapt the work, but must recognize the authors and the South African \\ Journal of Animal Science.
}

\begin{abstract}
The study investigated the effects of probiotic bacteria (Lactobacillus reuteri ZJ625, Lactobacillus reuteri VB4, Lactobacillus salivarius ZJ614, and Streptococcus salivarius NBRC13956) administered as direct-fed microorganisms on growth performance and blood parameters of weaned piglets. Forty-five weaned piglets were divided into five treatments: antibiotic (PC), no antibiotic and no probiotic (NC), probiotic (P1), probiotic (P2), and combination of probiotics (P3). Fecal and ileum samples were collected for microbial count analysis. Blood samples were also collected from the animals at the end of the trial for the hematological and biochemical analysis and the ability of the probiotics to stimulate immunoglobulin $\mathrm{G}$ (IgG). Supplementation of probiotics had no effect on feed intake (FI). However, average daily weight gained (ADG) in the P3 treatment was higher than in other treatments and lowered the value of feed conversion ratio (FCR) of weaned piglets. Microbial count of fecal samples did not differ in all the treatments while ileum samples had lower enteric bacteria in $\mathrm{P} 3$ treatment when compared to other treatments. Concentration of albumin, globulin, neutrophils and basophils were higher in the NC treatment when compared to other treatment groups. The IgG concentration was highest in P3 compared to other treatments. Results suggested that probiotics have beneficial effects on growth performances, blood parameters, and IgG stimulation of weaned piglets. This advocates that probiotics will offer a significant benefit in pig farming by reducing the risk of post weaning diarrheal syndromes, and therefore enhance pig industry's economy.
\end{abstract}

Keywords: Blood chemistry, feed conversion ratio, immunoglobulin G, post-weaning diarrheal syndromes, probiotics

\#Corresponding author: Aiyegoroo@arc.agric.za

\section{Introduction}

Weaning transition is a complicated phase in pig production because piglets are weaned from the ages of three to four weeks old (Moeser et al., 2007). During this phase, the piglets have to cope with unexpected hasty separation from the sows, mixed with other clutters in a strange environment, and their diet changes from easily digestible (milk) to less digestible (solid) food (Lalles, 2007). Weaning is a very critical period in pig production, as it may result in digestive disturbance, which causes growth set-back, and low feed intake in piglets. This can ultimately result in mortality in some cases (Hacin et al., 2008). This transition period is frequently linked with high occurrence of Post-Weaning Diarrheal Syndromes (PWDS), which is triggered by potential enteric pathogens such as Escherichia coli (E. coli) (Rist et al., 2013). PostWeaning Diarrheal Syndrome is a multifactorial disorder that arises after weaning, but is characterized by the proliferation of Enterotoxigenic E. coli (ETEC) in the gut (Tang et al., 2009). Once PWDS outbreaks occur in farms, the morbidity may be over $50 \%$ among the weaned piglets (Laine et al., 2008) and this eventually results in huge economic losses in the pig industry (Kiers et al., 2003).

Antibiotics have been added in the diet of just weaned piglets in order to reduce the occurrence of diarrhoea, while at the same time promoting growth performance (Thu et al., 2011). However, in some European countries, antibiotics have been legislatively and voluntarily banned for use in pigs' diet as growth promoters (Pluske et al., 2002). This is because of the concerns about antibiotic residues in animal food products and the development of bacterial resistance to antibiotics; hence, development for alternative to antibiotics is necessary. Probiotics are potential alternatives (Gu et al., 2006); probiotics are non-toxic live microorganisms that are normally used as animal feed additives. They produce beneficial effect to equilibrate 
intestinal flora of an animal (Yirga, 2015). Probiotics compete with harmful gut flora, stimulate the immune system of the animal, and increase its resistance to infectious agents in order to promote growth (Kritas \& Morrison, 2005); and have been reported to be able to lower blood cholesterol (Ooi \& Liong, 2010). Probiotics execute these health benefits by reducing metabolic reactions that produce toxic substances, produce vitamins and anti-microbial compounds such as bacteriocins (Hemaiswarya et al., 2013).

The most commonly used probiotics microorganisms belong to lactic acid bacteria (LAB), such as Lactobacillus spp., Bifidobacterium spp., and Enterococcus spp. (Ljungh \& Wadstrom, 2006), and Streptococcus spp. These bacteria are normally isolated from intestinal microflora of the intended species and selected based on conditions such as resistance to stomach acids, bile salts, ability to colonize the intestinal harmful microorganisms (Cho et al., 2011). Thus, the objective of this study was to test the efficacy of putative probiotic strains of Lactobacillus reuteri ZJ625, Lactobacillus reuteri VB4, Lactobacillus salivarius ZJ614, and Streptococcus salivarius NBCR 13956 on growth performance, hematological and biochemical parameters, and antibody stimulation on weaned piglets.

\section{Materials and Methods}

Procedures involved in this study complied with all the relevant legislation regarding the protection of animal welfare and were approved by the Agricultural Research Council (ARC), Animal Production Institute (API) Ethics Committee (APIEC13/008).

Probiotic bacteria were isolated and characterized from the gastro-intestinal tracts (GIT) of eight piglets of South African Windsnyer pig breed. These pigs have proven records of tolerance to diseases, high weaning rates and low incidences of post weaning diarrhoea (PWD). Combinations of both the molecular sequencing (16S ribosomal RNA) and analytical profile index (API® Biomerieux, SA) methods were used to identify the probiotics. The probiotics were prepared in MRS agar slants and stored in ultra-low-freezer. Lactobacillus reuteri ZJ625, Lactobacillus reuteri VB4, Lactobacillus salivarius ZJ614 and Streptococcus salivarius NBRC 13956 probiotic strains were revived by inoculation in Man, Rogosa and Sharpe broth (MRS; Oxoid, England). The probiotics were then grown in anaerobic jars with $\mathrm{CO}_{2}$-generating kits (Anaerogen; Thermofisher, UK) at $37{ }^{\circ} \mathrm{C}$ for $24 \mathrm{hr}$. The revived probiotic strains were inoculated with the appropriate culture at $1 \%(\mathrm{v} / \mathrm{v})$ and grown anaerobically in MRS broth overnight following the procedure described by Casey et al. (2007).

The study was carried out at the Agricultural Research Council (ARC) Gastrointestinal Microbiology and Biotechnology Unit and Pig Nutrition Unit in Gauteng, South Africa. Weaned piglets $(n=45)$, of which 23 were commercial (large white $\mathrm{x}$ landrace) breed piglets and 22 were South African indigenous Windsnyer breed piglets, entered the study at four weeks old, at a weight range of 7.5 to $9.5 \mathrm{~kg}$ and were in a trial for a period of 30 days. Pigs were kept individually in a temperature-controlled building in flat deck type cages (1.5 $x 1.0 \mathrm{~m}$ ) with perforated metal floor, equipped with self-feeders and automatic water nipple; and piglets had unrestricted access to water for the whole trial period.

The piglets were randomly assigned to five treatments ( 9 per treatment). Treatment 1 : diet containing no antibiotic and no probiotic (NC); Treatment 2: diet containing antibiotic - no probiotic (PC); Treatment 3: diet supplemented with probiotic (L. reuteri ZJ625) (P1); Treatment 4: diet supplemented with probiotic (S. salivarius NBRC13956) (P2); and Treatment 5: diet supplemented with combination of probiotics (L. reuteri ZJ625, L. reuteri VB4, L. salivarius ZJ614 and S. salivarius NBRC 13956) (P3). L. reuteri VB4 and L. salivarius ZJ614 have been tested and confirmed in our previous studies (forthcoming) to have probiotics properties; hence, in this study they are combined with newly tested probiotics to confirm synergy when all are combined together. The diet used in these experiments was in the form of pellets and was formulated to provide the nutrient requirements regardless of treatments, although for positive control group, in-feed antibiotic lyncospectin was added. The ingredients and composition of the experimental diets are presented in Table 1 and viable cell counts of probiotics used in the study are presented in Table 2.

The piglets were supplied with sufficient feed and water through self-feeders. Feed intake (FI) and body weight were monitored and measured weekly to determine the average daily gain (ADG), feed intake $(\mathrm{FI})$, and feed conversion ratio (FCR). The $\mathrm{FI}$ of pigs was recorded once a week by offering a weighed quantity of feed and weighing their residues, while the ADG was calculated by subtracting the initial body weight from the final body weight and thereafter divided by the number of feeding days on trial. The FCR for each piglet was calculated by dividing feed intake by the body weight gain. Piglets in a probiotic treatment were administered with $10 \mathrm{~mL}$ of 24 hour old culture once a week, orally using a syringe. Mortalities and morbidities were monitored, noted, and subjected to post mortem examination. 
Table 1 Nutritional levels of experimental diets (Meadow weaner feeds South Africa)

\begin{tabular}{lc}
\hline Nutrients & $\mathbf{g} / \mathbf{k g}$ \\
\hline Protein & 180 \\
Fat & 25 \\
Fibre & 50 \\
Dry matter & 880 \\
Calcium & 10 \\
Total lysine & 11 \\
\hline
\end{tabular}

Table 2 Average viable cell counts of probiotics administered to piglets

\begin{tabular}{lc}
\hline Probiotic & Concentration (cfu/mL) \\
\hline L. reuteri ZJ625 & $6.8 \times 10^{9}$ \\
L. reuteri VB4 & $5.5 \times 10^{10}$ \\
L. salivarius ZJ614 & $2.6 \times 10^{10}$ \\
S. salivarius NBRC13956 & $2.9 \times 10^{10}$ \\
\hline
\end{tabular}

A day after oral administration of probiotics, $10 \mathrm{~g}$ of fresh fecal samples were collected weekly from each piglet, for bacterial enumeration and stored at $-80^{\circ} \mathrm{C}$. At the end of the trial (30 days), pigs were slaughtered and a segment of ileum was removed and placed in a sterile tube containing $9 \%(\mathrm{v} / \mathrm{v})$ normal saline for bacterial enumeration. Collected fecal and ileum samples were serially diluted from $10^{-1}$ to $10^{-8}$. Dilutions were subsequently plated in triplicate on selective agar media for enumeration of target bacterial strains. Total bacteria, enteric and lactic acid bacteria (LAB) were enumerated using nutrient agar (Oxoid, England), violet red bile agar (VRB; Oxoid, England) and Man, Rogosa and Sharpe agar (MRS; Oxoid, England) respectively, following the traditional method of An et al. (2008). Each plate was incubated at $37^{\circ} \mathrm{C}$ for 24 hour, anaerobically for $L A B$ and aerobically for enteric and total bacteria. Colonies were counted using automated colony counter (Ben-art product, USA). Results were presented as the average of the three dilutions at base 10- logarithm colony forming unit per $\mathrm{mL}(\mathrm{cfu} / \mathrm{mL})$.

At the end of the trial, approximately $20 \mathrm{~mL}$ of blood were collected from sacrificed piglets, for the analysis of hematological, biochemical and $\operatorname{lgG}$ stimulation. Blood samples collected into EDTA (BD Diagnostics, pre-analytical systems, Midrand, USA) were transported on ice to the Diagnostic Laboratory, for the determination of selected hematological parameter analysis. The hematological parameters analyzed included red blood cells (RBC) (hemoglobin and Hematocrit), white blood cells (WBC) (neutrophils, monocytes, lymphocytes, eosinophils, basophils) and platelets. In addition, biochemical parameters analyzed include total serum protein, albumin, globulin, cholesterol and glucose.

The preparation of serum was carried out using the method of Szabo et al. (2009). Aliquots $(2 \mathrm{~mL})$ of blood samples collected into serum bottles were transferred into sterile $5 \mathrm{~mL}$ microcentrifuge tubes and centrifuged at $1200 \mathrm{rpm}$ for $15 \mathrm{~min}$ using Sorvall MC-12V microcentrifuge (American Laboratory trading, Inc., USA). The fraction sera were later aspirated using Pasteur pipettes (Sigma-Aldrich, Johannesburg, South Africa) into sterile $5 \mathrm{~mL}$ centrifuge tubes and used within 12 hour of preparation. Swine serum samples were diluted $(1: 35,000)$ and concentrations of IgG were quantified using Pig IgG enzyme-linked immunosorbent assay (ELISA) kits (Bethyl laboratories, Inc.; USA), following manufacturer's instructions. A Multiskan GO Microplate Spectrophotometer (Thermofisher Scientific, USA) was then used to measure the substrate absorbance at a wavelength of $450 \mathrm{~nm}$. The results obtained $(\mathrm{ng} / \mathrm{mL})$ were multiplied by $10^{-5}$ dilution factor and expressed as $\mathrm{mg} / \mathrm{mL}$. In each experiment serial dilution of standard samples were tested in order to obtain calibration curve, which was then computer-adjusted (with Fin graph software program). From this calibration curve, values of unknown IgG concentration were calculated. 
The data was submitted to one-way analysis of variance (ANOVA), comparing five treatments as main effects, using SAS statistical software (SAS 9.3) (2003). Means were separated using Tukey's Test and differences were considered significant at $P \leq 0.05$.

\section{Results and Discussion}

In all treatment groups, no significant difference was observed $(P>0.05)$ for FI (Table 3$)$. The ADG on P3 treatment group was higher $(P \leq 0.05)$ as compared to other treatment groups and FCR was better in P3 treatment group.

Table 3 Growth performance of piglets based on different treatments group

\begin{tabular}{lcccccc}
\hline \multirow{2}{*}{ Parameters } & \multicolumn{5}{c}{ Treatment } & \multirow{2}{*}{ P-value } \\
\cline { 2 - 6 } & NC & PC & P1 & P2 & P3 & \\
\hline FI (kg/d) & 0.83 & 0.81 & 0.82 & 0.86 & 0.80 & 0.57 \\
ADG (kg/d) & $0.40^{\mathrm{c}}$ & $0.41^{\mathrm{c}}$ & $0.40^{\mathrm{c}}$ & $0.47^{\mathrm{b}}$ & $0.57^{\mathrm{a}}$ & 0.04 \\
FCR & $2.10^{\mathrm{a}}$ & $2.00^{\mathrm{a}}$ & $2.10^{\mathrm{a}}$ & $1.90^{\mathrm{a}}$ & $1.40^{\mathrm{b}}$ & 0.03 \\
\hline
\end{tabular}

${ }^{\mathrm{a}-\mathrm{c}}$ Mean values within a row with different superscripts are significantly different $(P \leq 0.05)$

$\mathrm{ADG}=$ Average daily gain, $\mathrm{FI}=$ Feed Intake, $\mathrm{FCR}=$ Feed conversion ratio, $\mathrm{NC}=$ Negative control, $\mathrm{PC}=\mathrm{Positive} \mathrm{Control}$ $\mathrm{P} 1=$ L. reuteri ZJ625, P2 $=S$. salivarius NBRC13956, P3 = Combination of probiotics $(L$. reuteri ZJ625, $L$. reuteri VB4, $L$. salivarius ZJ614 and S. salivarius NBRC13956).

Table 4 shows effect of probiotic bacteria on biochemical parameters. No significant difference $(P$ $>0.05)$ was observed in all treatments when total serum protein, cholesterol and glucose were measured in the experimental animals. The concentration of albumin and globulin showed higher significant difference $(P$ $\leq 0.05$ in NC treatment group as compared to other treatment groups.

Table 4 Biochemical parameters of piglets based on different treatments groups

\begin{tabular}{lcccccc}
\hline \multirow{2}{*}{ Parameters } & \multicolumn{7}{c}{ Treatments } & P-value \\
\cline { 2 - 6 } & NC & PC & P1 & P2 & P3 & 0.69 \\
TSP (g/L) & 54.1 & 55.1 & 58.9 & 54.2 & 60.5 & 0.03 \\
ALB (g/L) & $47.1^{\mathrm{a}}$ & $45.6^{\mathrm{b}}$ & $44.9^{\mathrm{b}}$ & $43.0^{\mathrm{b}}$ & $38.5^{\mathrm{c}}$ & 0.05 \\
$\mathrm{GLO}(\mathrm{g} / \mathrm{L})$ & $65.5^{\mathrm{a}}$ & $63.3^{\mathrm{b}}$ & $60.9^{\mathrm{b}}$ & $59.2^{\mathrm{c}}$ & $58.4^{\mathrm{c}}$ & 0.99 \\
$\mathrm{CHO}(\mathrm{mmol} / \mathrm{L})$ & 2.10 & 2.03 & 2.27 & 2.13 & 2.08 & 0.94
\end{tabular}

\footnotetext{
${ }^{\mathrm{a}-\mathrm{c}}$ Mean values within a row with different superscripts are significantly different $(P \leq 0.05)$

$\mathrm{NC}=$ Negative control, PC $=$ Positive Control P1 $=$ L. reuteri ZJ625, P2 = S. salivarius NBRC13956, P3 = Combination of probiotics (L. reuteri ZJ625, L. reuteri VB4, L. salivarius ZJ614 and S. salivarius NBRC13956).

$\mathrm{TSP}=$ Total serum protein, $\mathrm{ALB}=$ Albumin, $\mathrm{GLO}=$ Globulin, $\mathrm{CHO}=$ Cholesterol, $\mathrm{GLU}=$ Glucose
}

Table 5 shows effect of probiotic bacteria on hematological parameters. No significant difference $(P$ $>0.05$ ) observed in all treatments except for segmented neutrophils and basophils. Segmented neutrophils and basophils had higher concentration $(P \leq 0.05)$ in NC treatment group as compared to other treatment groups. 
Table 5 Hematological analysis of piglets based on different treatment groups

\begin{tabular}{|c|c|c|c|c|c|c|}
\hline \multirow{2}{*}{ Parameters } & \multicolumn{5}{|c|}{ Treatments } & \multirow[t]{2}{*}{$P$-value } \\
\hline & NC & PC & P1 & $\mathbf{P 2}$ & P3 & \\
\hline $\mathrm{Hb}(\mathrm{g} / \mathrm{L})$ & 140 & 145 & 137 & 136 & 131 & 0.15 \\
\hline \multicolumn{7}{|l|}{ Cells/L (10E9) } \\
\hline $\mathrm{Ht}$ & 0.44 & 0.45 & 0.44 & 0.43 & 0.40 & 0.09 \\
\hline Wcc & 19.4 & 21.1 & 15.2 & 14.7 & 21.1 & 0.13 \\
\hline Sn & $14.4^{\mathrm{a}}$ & $9.5^{\mathrm{b}}$ & $7.33^{b}$ & $4.92^{\mathrm{C}}$ & $4.81^{\mathrm{C}}$ & 0.01 \\
\hline $\mathrm{Bn}$ & 0.00 & 0.38 & 0.58 & 0.00 & 1.71 & 0.34 \\
\hline Lym & 8.58 & 9.30 & 6.41 & 8.87 & 7.65 & 0.75 \\
\hline Mon & 1.14 & 0.66 & 0.74 & 0.68 & 0.71 & 0.39 \\
\hline Eos & 0.48 & 0.20 & 0.66 & 0.11 & 0.20 & 0.16 \\
\hline Bas & $0.16^{\mathrm{a}}$ & $0.10^{\mathrm{a}}$ & $0.07^{b}$ & $0.14^{\mathrm{a}}$ & $0.04^{\mathrm{C}}$ & 0.05 \\
\hline Plt & 311 & 430 & 380 & 430 & 420 & 0.71 \\
\hline
\end{tabular}

\footnotetext{
${ }^{a-c}$ Mean values within a row with different superscripts are significantly different $(P \leq 0.05)$

$\mathrm{NC}=$ Negative control, PC $=$ Positive Control P1 $=$ L. reuteri ZJ625, P2 $=$ S. salivarius NBRC13956, P3 = Combination of probiotics (L. reuteri ZJ625, L. reuteri VB4, L. salivarius ZJ614 and S. salivarius NBRC13956).

$\mathrm{Wcc}=$ White cell count, $\mathrm{Sn}=$ Segmented neutrophils, $\mathrm{Bn}=$ Band neutrophils, Lym $=$ Lymphocytes, Mon $=$ Monocytes, Eos $=$ Eosinophil, Bas $=$ Basophil, Plt $=$ Platelet
}

Several studies on probiotics have been published in recent years, suggesting that probiotics offer beneficial nutrition and protection against pathogens for animals and humans, particularly in the pig industry (Chaucheyras-Durand and Durand, 2010; Ezema, 2013; Devi and Kim, 2014). In the present study, no significant difference was observed for FI between the treatment groups (Table 3). However, ADG on the P3 treatment group (combination of probiotics) was higher $(P \leq 0.05)$ as compared to other treatment groups and resulted in a drastic improvement in FCR in the P3 treatment group. The FCR of this P3 group improved from $2 \mathrm{~kg}$ of feed consumed for $1 \mathrm{~kg}$ of weight gain to $1.4 \mathrm{~kg}$ of feed consumed for $1 \mathrm{~kg}$ of weight gain, which is an improvement of $30 \%$ in efficiency. The extrapolation from these results is that the producer would have invested less money per kilo of weight when the pig reaches slaughter weight. Lojanica et al. (2010) reported similar results when evaluated effects of probiotic Enterococcus faecium DSM 7134 in the weaned piglets nutrition. They found that piglets fed E. faecium had greater daily weight gain and improved feed conversion ratio. Vrotniakiene \& Jatkauskas (2013) also reported improvement in both daily weight gain and feed conversion ratio, when pig diet is supplemented with Enterococcus-based probiotics. These results demonstrate that supplementation of a combination of probiotics has potential to be used as a dietary supplementation in weaned piglets.

A properly balanced gut microbiota has the ability to positively affect the integrity of the intestinal barrier against colonization by pathogens (Jandhyala et al., 2015), through its protective and metabolic function and can stimulate the immune system in an anti-inflammatory manner ( $\mathrm{Wu}$ and $\mathrm{Wu}, 2012$ ). However, physiological and/or psychological stresses such as weaning lead to dysfunctional of gut microbial composition (McLamb et al., 2013). By supplementing probiotics to the piglets feeding during post weaning, there is a potential to modulate intestinal microbial diversity by improving the establishment of beneficial bacterial populations for health and reducing pathogenic bacteria such as enterotoxigenic E. coli (Brousseau, et al., 2015). In this study, the supplementation of probiotics did not reduce enteric bacterial counts nor increased lactic acid bacterial (LAB) counts in fecal samples (Figure 1). These results are similar to those reported by Giang et al. (2011), where how probiotics supplementation affects performance, nutrient digestibility and fecal microflora in growing-finishing pigs was evaluated. The authors reported that inclusion of Bacillus in pig diet did not affect fecal microflora. However, Figueiredo-Silva et al. (2010) found different results, while studying probiotics and antibiotics as additives for sows and piglets during nursery phase. They found that the prevalence of $E$. coli associated with pathogenic groups was lower with the use of probiotics. $\mathrm{Hu}$ et al. (2014) also reported that probiotic supplementation on diet of weaned piglets reduced $E$. coli counts in fecal samples. 


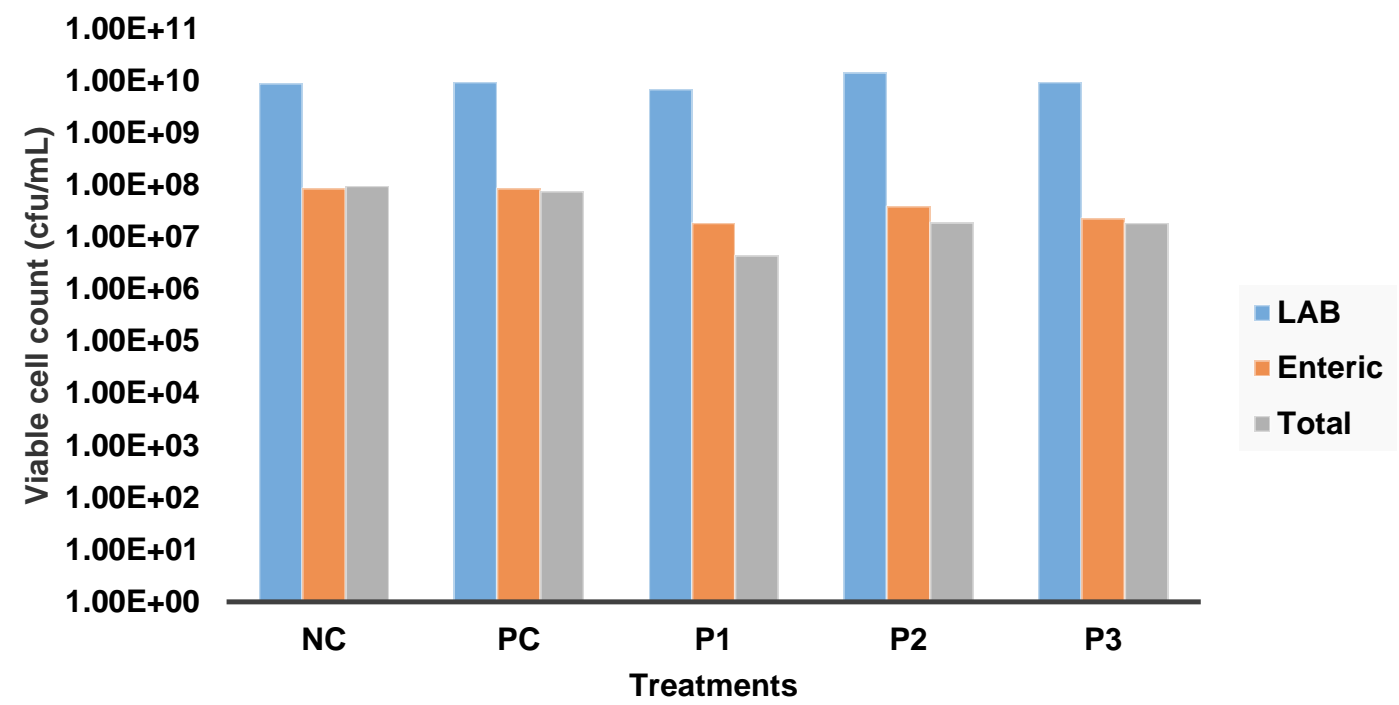

Figure 1 Microbial enumeration of lactic acid, enteric and total bacteria from piglets fecal samples

$\mathrm{NC}=$ Negative control, $\mathrm{PC}=$ Positive Control P1 $=$ L. reuteri ZJ625, P2 $=$ S. salivarius NBRC13956, P3 = Combination of probiotics (L. reuteri ZJ625, L. reuteri VB4, L. salivarius ZJ614 and S. salivarius NBRC13956).

From ileum samples counts, no significant difference was observed in all treatment groups, with an exception of P3 treatment group regarding cfu/mL of lactic acid bacteria, enteric bacteria and total bacteria. P3 treatment group had lower $\mathrm{cfu} / \mathrm{mL}$ of enteric bacteria as compare to lactic acid bacteria and total bacteria (Figure 2). It has been reported that probiotic bacteria maintain normal gut microflora in two ways: competitive exclusion and antagonism. Once probiotic bacteria reach the gut and established themselves, they may produce bactericidal compounds (bacteriocins) such as organic acids, hydrogen peroxide, and lactoferrin. These substances reduce the $\mathrm{pH}$ in the gut and this creates an environment where pathogenic bacteria cannot grow (Kamada, 2013).

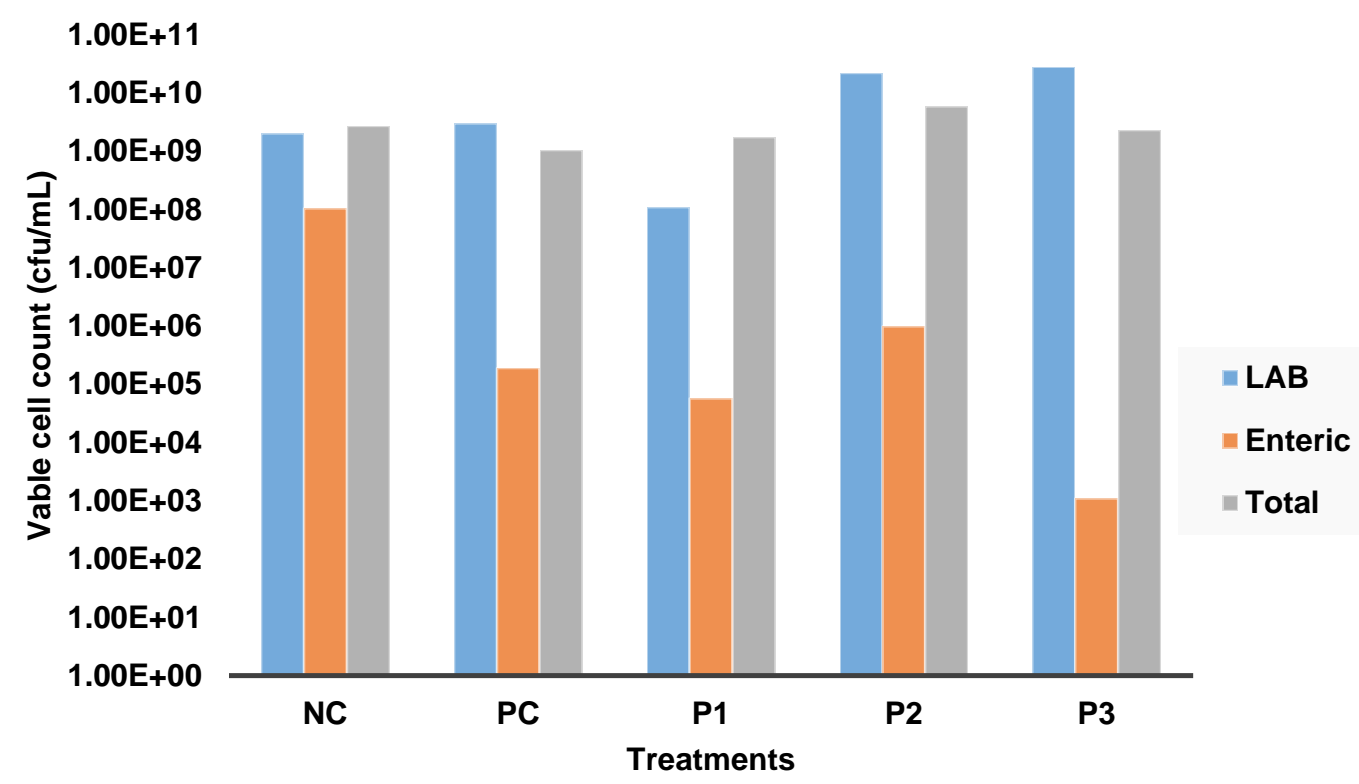

Figure 2 Microbial enumeration of lactic acid, enteric and total bacteria from piglets ileum samples.

$\mathrm{NC}=$ Negative control, $\mathrm{PC}=$ Positive Control P1 $=$ L. reuteri ZJ625, P2 $=$ S. salivarius NBRC13956, P3 = Combination of probiotics (L. reuteri ZJ625, L. reuteri VB4, L. salivarius ZJ614 and S. salivarius NBRC13956). 
These results are consistent with results of Mallo et al. (2010), who found that the addition of Enterococcus faecium to diet improves the intestinal microbiota of the piglets. In their study, they observed that piglets receiving the probiotic bacteria in the diet had significantly higher cfu/mL of lactic acid bacteria in the ileum while the $\mathrm{cfu} / \mathrm{mL}$ of $E$. coli was significantly lower. Enterobacteria are potential intestinal pathogens when an imbalance of the intestinal microflora occurs. It has been reported that a decrease of $E$. coli in probiotic-fed piglets led to improved LAB/E coli ratio, a suggested indicator of gut health (Dong et al., 2013). The decrease in enteric bacteria in ileum samples of piglets fed a combination of probiotics (P3) indicates improved gut health.

A well-balanced gut microflora is able to affect the integrity of the intestinal barrier against colonization through its protective and metabolic function and can stimulate the immune system in an antiflammatory manner. However, physiological or psychological stresses caused by weaning process compromise gut microbiota and results to intestinal dysfunction. Probiotics are mostly applied on this kind of situation, in order to re-establish the gut microbial balance (Ahmed et al., 2014). The results from this study indicates that supplementation of probiotics in weaned piglets feed enhance the immune system.

Results on Table 4 show the probiotics effect on blood biochemistry parameters of the slaughtered piglets. No significant difference was observed for parameters such as total serum protein, cholesterol and glucose among all treatment groups. It was only observed for globulin and albumin; negative control group had high concentration of albumin and globulin. These results are similar to those observed by Busanello et al. (2015), who concluded that high concentration of albumin and globulin indicate the presence of infection and dehydration. This suggest that piglets in the control treatment group were more exposed to infections as compared to piglets in the probiotic treatment groups. On the contrary, Kumar et al. (2012) reported no significant difference observed in the concentration of albumin and globulin in all treatment groups.

Findings of probiotic effect on hematology of slaughtered piglets' blood are shown on Table 5. No significant difference was observed amongst treatment groups regarding hematology parameters, except for segment neutrophils and basophils. Segment neutrophils and basophils had higher (concentration in the control treatment group than in probiotic groups. These findings are similar to those of Nurliyani and Marsetyawan (2011) who reported that rat fed probiotic fermented goat milk had lower levels of neutrophils and basophils than rats in control treatment. Moreover, high levels of neutrophils and basophils indicates occurrence of infection. These findings clearly indicate that piglets in probiotic groups were in a healthier state as compared to those in the control treatment group. However, Al-Saiady et al. (2010) did not find significant improvement on hematology parameters from the use of probiotics.

The Figure 3 shows the effect of probiotics on IgG stimulation in piglets. Piglets fed probiotics had higher concentration of IgG than piglets fed positive and negative control feed. This significant increase of IgG in the probiotic group could be due to the persistent of probiotic bacteria in the intestinal tract and acting as immune adjuvant to the immune system and therefore stimulating IgG production (Naqid et al., 2015).

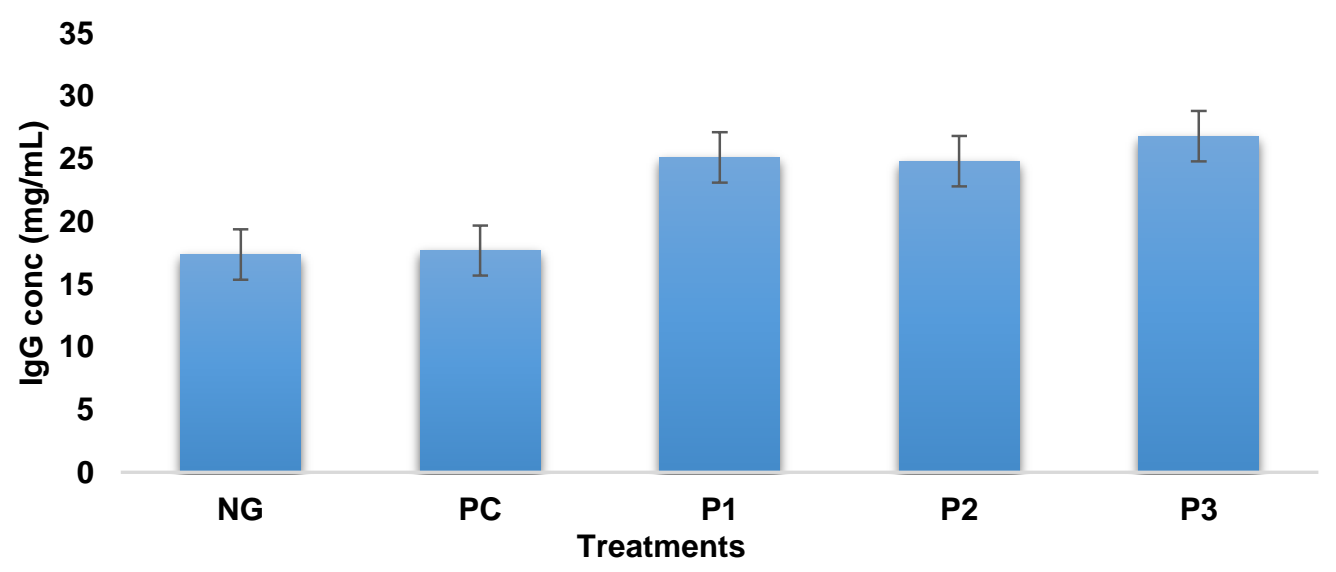

Figure 3 Effect of different treatments on Immunoglobulin G (IgG) stimulation of piglets

$\mathrm{NC}=$ Negative control, $\mathrm{PC}=$ Positive Control P1 $=$ L. reuteri ZJ625, P2 $=$ S. salivarius NBRC13956, P3 = Combination of probiotics (L. reuteri ZJ625, L. reuteri VB4, L. salivarius ZJ614 and S. salivarius NBRC13956). 
These results are in accordance with results of Yu et al. (2004). Yu and his co-authors evaluated the effect of probiotics and selenium combination on the immune and blood cholesterol concentration of pig. In their findings, they reported that piglets fed probiotics showed higher concentration of IgG than piglets fed treatment without probiotics. On contrary, Chen et al. (2005) reported different result when studied the effect of dietary probiotics on growth performances, nutrient digestibility, blood characteristics and fecal noxious gas content in growing pigs. In their findings, they reported IgG concentration was not affected by dietary treatment of complex probiotics. These results suggest that piglets administered with probiotics were healthier than those getting antibiotics. The findings correlates with challenges piglets face during weaning phase; such as low feed intake, stressful environment and weight loss. Thus, piglets in NC presented poor immune system.

\section{Conclusions and Recommendations}

From this study, the application of a probiotics blend in piglets clearly revealed that probiotics have great potential as alternatives to antibiotics. Although different probiotics strains, even of the same species may have different metabolic effects; which in turn affect growth performances, microbial count and blood parameters of piglets differently. Moreover, probiotics may be used to control post weaning diarrheal syndromes during weaning period. However, the mechanism of actions of probiotics is largely unknown. Hence, it is of utmost importance for more research to be carried out in order to elucidate the mechanism of probiotic actions, to provide more rigid recommendations about probiotics strains and their optimal performance levels. Additionally, studies of shelf life, supplementation stages, alternatives to oral administration, dose size, effect on different pig breeds and gender are also required.

\section{Acknowledgements}

The authors would like to acknowledge the Agricultural Research Council - Animal Production Institute, Irene, South Africa; the Red Meat Research \& Development South Africa (RMRDSA) and the South Africa Medical Research Council for supporting this work.

\section{Authors' Contributions}

ZCD conducted the research for her MSc studies and drafted the manuscript, RLSL helped with animal trials and laboratory work, OAA and AIO conceived, designed and supervised the research. Finally, all authors commented on early and final versions of the manuscript.

\section{Conflict of Interest Declaration}

The authors have declared that there is no conflict of interests.

\section{References}

Ahmed, S.N., Hoon, J., Mun, H.S. \& Yang, C.J., 2014. Evaluation of Lactobacillus and Bacillus-based probiotics as alternatives to antibiotics in enteric microbial challenged weaned piglets. Afr. J. Microbiol. Res. 8, 96-104.

Al-Saiady, M.Y., 2010. Effect of probiotic bacteria on immunoglobulin G concentration and other blood components of new calves. J. Anim. Vet. Adv. 9, 604-609.

An, B.K., Cho, B.L., You, S.J., Paik, H.D., Chang, H.I., Kim, S.W., Yun, S.W., Yun, C.W. \& Kang, C.W., 2008. Growth performance and antibody response of broiler chicks fed yeast derived $\beta$-glucan and single-strain probiotics. Asian-Aust. J. Anim. Sci. 21, 1027-1032.

Balasubramanian, B., Li, T. \& Kim, I.H., 2016. Effect of supplementing growing-finishing pig diets with bacillus spp. Probiotic on growth performance and meat-carcass grade quality traits. R. Bras. Zootec. 45, 93-100.

Brousseau, J.P., Talbot, G., Beaudoin, F., Lauzon, K., Roy, D. \& Lessard, M., 2015. Effect of probiotics Pediococcus acidilactici strain MA18/5M and Saccharomyces cerevisiae subsp. boulardii strain SB-CNM I-1079. On faecal and intestinal microbiota of nursing and weanling piglets. J. Anim. Sci. 93, 5313-5326.

Busanello, M., Pozza, M.S.S., Pozza, P.C., Nunes, R.V., Chambo, A.P.S. \& Eckstein, I.I., 2015. Probiotics: viable and inactivated cells on the performance, microflora and blood parameters of piglets. Rev. Bras. Saúde. Prod. Anim. 16, 387-396.

Casey, P.G., Gardner, G.E., Casey, G., Bradshaw, B., Lawler, P.G., Lynch, P.B., Leonard, F.C., Stanton, C., Ross, P.R., Fitzgerald, G.F. \& Hill, C., 2007. A five-strain probiotic combination reduces pathogens shedding and alleviates disease signs in pigs challenged with Salmonella enteric Serova Typhimurium. Appl. Environ. Microbiol. 73, 18581863.

Chaucheyras-Durand, E. \& Durand, H., 2010. Probiotics in animal nutrition and health. J. Benef. Microbes 1, 3-9.

Chen, Y.J., Son, K.S., Min, B.J., Cho, J.H., Kwon, O.S. \& Kim, I.H., 2005. Effect of dietary probiotic on growth performance, nutrients digestibility, blood characteristics and fecal noxious gas content in finishing pigs. AsianAustralas. J. Anim. Sci. 19, 587-592.

Cho, J.H., Zhao, P.Y. \& Kim, I.H., 2011. Probiotics as dietary additives for pigs: A review. J. Anim. Vet. Adv. 10, 21272134. 
Devi, S.M. \& Kim, I.H., 2014. Effect of medium chain fatty acids (MCFA) and probiotic (Enterococcus faecium) supplementation on the growth performances, digestibility and blood profile in weaned pigs. Vet. Med. 59, 572535.

Dong, X., Zhang, N., Zhou, M., Tu, Y., Deng, K. \& Diao, Q., 2013. Effect of dietary probiotics on growth performances, fecal microbiota and serum profiles in weaned piglets. Anim. Prod. Sci. 54, 1-6.

Dowarah, R., Verma, A.K., Agarwal, N., Patel, B.H.M. \& Singh, P., 2017. Effect of swine based probiotic on performance, diarrhoea scores, intestinal microbiota and gut health of grower-finisher crossbred pigs. Livest. Sci. 195, 74-79.

Ezema, C., 2013. Probiotics in animal production: A review. J. Vet. Med. Anim. Health 5, 308-316.

Figueiredo-Silva, M.L.F., de Freitas-Lima, J.A., de Souza-Cantarelli, V., de Oliveira-Amaral, N., Zangeronimo, M.G. \& Fialho, E.T., 2010. Probiotics and antibiotics as additives for sows and piglets during nursery phase. R. Bras. Zootec. 39, 2453-2459.

Giang H.H., Viet, T.Q., Ogle, B. \& Lindberg, J.E., 2011. Effect of supplementation of probiotics on the performance, nutrient digestibility and fecal microflora in growing-finishing pigs. Asian-Australas. J. Anim Sci. 24, 655-661.

Gu, X., Li, D., Lu, W., Piao. X. \& Chen, X., 2006. Screening of Bacillus strains as potential probiotics and subsequent confirmation of the in vitro effectiveness of Bacillus subtilis MA139 in pigs. Antonie. Van. Leeuwenhoek. 90, 139146.

Hacin, B., Rogelj, T. \& Matijasic, B.B., 2008. Lactobacillus isolates from weaned piglets mucosa with inhibitory activity against common porcine pathogens. Folia Microbial 53, 569-579.

Hemaiswarya, S., Raja, R., Ravikumar, R. \& Carvalho, I.S., 2013. Mechanism of action of probiotics. Braz. Arch. Biol. Technol. 56, 113-119.

Hu, Y., Dun, Y., Li, S., Zhao, S., Peng, N. \& Liang, Y., 2014. Effects of Bacillus subtilis KN-42 on growth performance, diarrhea and faecal bacterial flora of weaned piglets. Asian-Aust. J. Anim. Sci. 27, 1131-1140.

Jandhyala, S.M, Talukdar, R., Subramanyam, C., Vuyyuru, H., Sasikala, M. \& Reddy, D.N., 2015. Role of the normal gut microbiota. World. J. Gastroenterol 21:8787-8803.

Kamada, N., 2013. Control of pathogens and pathobionts by the gut microbiota. Nat. Immunol. 14, 685-690.

Kiers, J.L., Meijer, J.C., Nout, M.J.R., Rombouts, F.M., Nabuurs, M.J.A. \& Van der Meulen, J., 2003. Effect of fermented soybeans on diarrhea and feed efficiency in weaned piglets. J. Appl. Microbiol. 95, 545-552.

Kritas, S.K. \& Morrison, R.B., 2005. Evaluation of probiotics as a substitute for antibiotics in a large pig nursery. Vet. Rec. $156,447-448$.

Kumar, S., Verma, A.K., Mondal, S.K., Gupta, M., Patil, A.K. \& Lal Jangir, B., 2012. Effect of Saccharomyces cerevisiae feeding on serum biochemistry in early-weaned crossbred piglets. Vet. World 5, 663-666.

Laine, T.M., Lyttikäinen, T., Yliaho, M. \& Anntila, M., 2008. Risk factors for post-weaning diarrhea on piglets producing farms in Finland. Acta Vet. Scand. 50, 1-11.

Lalles, J.P., Bosi, P., Smidt, H. \& Stokes, C.R., 2007. Nutritional management of gut health in pigs around weaning. Proc. Nutr. Soc. 66, 260-268.

Ljungh, A. \& Wadstrom, T., 2006. Lactic acid bacteria as probiotics. Curr. Issues Intestinal Microbiol. 7, 73-90.

Lojanica, M., Manojlovic, M. \& Jeremic, D. \& Petronijevic, S., 2010. The effects of probiotic Enterococcus faecium DSM 7134 in the weaned pigs nutrition. Biotechnol. Anim. Husb. 26, 57-64.

Mallo, J.J., Rioperez, J. \& Honrubia, P., 2010. The addition of Enterococcus faecium to diet improves piglet's intestinal microbiota and performances. J. Livest. Sci. 133, 176-178.

McLamb, B.L., Gibson, A.L., Overman, E.L., Stahl, C. \& Moeser, A.J., 2013. Early weaning stress in pigs impairs innate mucosal immune responses to Entertoxigenic $E$. coli challenge and exacerbates intestinal injury and clinical disease. Plos. One. 18, 1-12.

Moeser, A.J., Ryan, K.A., Nighot, P.K. \& Blikslager, A.T., 2007. Gastrointestinal dysfunction induced by early weaning is attenuated by delayed weaning and mast cell blockade in pigs. Am. J. Physiol. Gastrointest. Liver. Physiol. 293, 413-421.

Naqid, I.A., Owen, J.P., Maddison, B.C., Gardner, D.S., Foster, N., Tchórzewska, M.A. \& La Ragione, R.M., 2015. Prebiotic and probiotic agents enhance antibody-based immune responses to Salmonella Typhimurium infection in pigs. Anim. Feed. Sci. Technol. 201, 57-65.

Nurliyani, E.H. \& Marsetyawan, H.S., 2011. Leukocytes count and lymphocytes proliferation of dinitrochlorobenzene sensitized rat supplemented with fermented goat milk. Int. J. Biol. Biomolec. Agric. Food Biotechnol. Eng. 5:357361.

Ooi, L.G. \& Liong, M.T., 2010. Cholesterol-lowering effects of probiotics and prebiotics: A review of in vivo and in vitro findings. Int. J. Mol. Sci. 11, 2499-2522.

Pluske, J.R., Pethick, D.W., Hopwood, D.C. \& Hampson, D.J., 2002. Nutritional influences on some major enteric bacterial diseases of pigs. Nutr. Res. 15, 333-371.

Rist, V. T.S., Weiss, E., Eklund, M. \& Mosenthin, R., 2013. Impact and activity on microbiota composition and activity in the gastrointestinal tract of piglets in relation to gut health: Anim. 7, 1067-1078.

SAS/STAT User's Guide, Version 9, $3^{\text {st }}$ printing, Volume 2. SAS Institute Inc, SAS Campus Drive, Cary, North Carolina 27513.

Szabo, I., Wieler, L.H., Teden, K., Scharek-Teden, L., Taras, D., Hensel, A., Appel, B. \& Nockler, K., 2009. Influence of a probiotic strain of Enterococcus faecium on Salmonella enterica Serovar Thyphimirium DT104 infection in a porcine animal infection model. Appl. and Environ. Microbiol. 75, 2621-2628.

Tang, Z., Yin, Y., Zhang, Y., Huang, R., Sun, Z., Li, T., Chu, W., King, X., Li, L., Geng, M. \& Tu, Q., 2009. Effects of dietary supplementation with an expressed fusion peptide bovine lactoferricin-lactoferrampin on performance, immune function and intestinal mucosal morphology in piglets weaned at age 21d. Br. J. Nutr. 101, 998-1005. 
Thu, T.V., Loh, T.C., Foo, H.L., Yaakub, H. \& Bejo, M.H., 2011. Effect of liquid metabolite combinations produced by Lactobacillus plantarum on growth performance, feces characteristics, intestinal morphology and diarrhea incidence in post-weaning piglets. Trop. Anim. Health. Prod. 43, 69-75.

Vrotniakiene, V. \& Jatkauskas, J., 2013. Effect of probiotics dietary supplementation on diarheoa incidence, fecal shedding of Escherichia coli and growth performances in post-weaned piglets. Vet. Med. Zoot. 63, 81-88.

Wu, H.J. \& Wu, E., 2012. The role of gut microbiota in immune homeostasis and autoimmunity. J. Gut. Microbes. 3, 4-14. Yirga, H., 2015. The use of probiotics in animal nutrition. J. Prob. Health. 3, 1-10.

Yu, L.T., Ju, C.C., Ju, J., Wu, H.L. \& Yen, H.T., 2004. Effect of probiotics and selenium combination on the immune and blood cholesterol concentration of pigs. J. Anim. Feed. Sci. 13, 625-634. 